Regards sur l'économie allemande

Bulletin économique du CIRAC

$70 \mid 2005$

Varia

\title{
Structures de la recherche : le centre de Jülich (FZJ) sur la voie de la réforme
}

\section{Marie-Hélène Pautrat}

\section{OpenEdition}

\section{Journals}

Édition électronique

URL : http://journals.openedition.org/rea/2013

DOI : 10.4000/rea.2013

ISBN : 978-2-8218-0836-2

ISSN : 1965-0787

\section{Éditeur}

CIRAC

\section{Édition imprimée}

Date de publication : 1 mars 2005

Pagination : 19-24

ISSN : 1156-8992

\section{Référence électronique}

Marie-Hélène Pautrat, « Structures de la recherche : le centre de Jülich (FZJ) sur la voie de la réforme », Regards sur l'économie allemande [En ligne], 70 | mars 2005, mis en ligne le 16 décembre 2008, consulté le 04 mai 2019. URL : http://journals.openedition.org/rea/2013 ; DOI : 10.4000/ rea.2013 


\section{Structures de la recherche : le centre de Jülich (FZJ) sur la voie de la réforme}

\section{Marie-Hélène Pautrat}

Le gouvernement Schröder a fait de la politique de recherche une priorité : il s'agit de conforter la place de l'Allemagne dans la recherche internationale et surtout de renforcer le système d'innovation, garant de la compétitivité du pays. Excellence scientifique, concentration des efforts sur des domaines jugés stratégiques et sur des secteurs technologiques-clefs constituent les principaux axes de cette stratégie. Cette politique encourage également l'organisation en réseau et le renforcement des liens entre organismes de recherche, enseignement supérieur et milieux économiques. Dans un système caractérisé par une forte diversité institutionnelle, il revient aux différents acteurs de mettre en pratique, chacun à sa manière, ces principes directeurs. La réforme conduite depuis 2001 dans les centres Helmholtz, à l'exemple ici du Forschungszentrum Jülich (FZJ), s'inscrit directement dans cette ambition politique de moderniser le système de recherche. Mais le FZJ n'est pas seulement le plus grand centre interdisciplinaire de recherche en Allemagne. Sa longue expérience de participation à des réseaux et programmes nationaux et internationaux en fait également un maillon important de la recherche européenne.

Fondé en 1956, le Forschungszentrum Jülich $\mathrm{GmbH}$ est un des quinze centres constituant l'association Hermann Helmholtz (Helmholtz-Gemeinschaft Deutscher Forschungszentren e.V., HGF), la principale organisation de recherche en Allemagne. II en est également l'un des plus importants avec 4350 salariés (1 280 chercheurs, 350 doctorants) et un budget de 360 millions $€$ en 2003. Le Bund et le Land de Rhénanie du Nord-Westphalie (NRW) détiennent respectivement $90 \%$ et $10 \%$ de son capital $(211,291$ millions $€)$. Le FZJ est composé de 37 instituts organisés en 12 départements, de 4 départements centraux, de 2 groupes de projets et héberge 2 entités de management de projets largement autonomes (Projektträger) En 2003, le centre comptait à son actif 1662 publications scientifiques, a organisé une cinquantaine de rencontres scientifiques et détenait des droits pour 8705 brevets, demandes de brevets ou modèles déposés $(1,9$ million $€$ en 2003).

\section{La Helmholtz-Gemeinschaft Deutscher Forschungszentren e.V. (HGF)}

Créée en 1995 pour succéder à la Arbeitsgemeinschaft der Großforschungseinrichtungen, la HGF constitue l'un des quatre piliers de l'organisation de la recherche publique allemande (budget $2003: 2,2$ milliards $€ ; 24000$ salariés). Elle regroupe aujourd'hui 15 centres, fondés pour la plupart dans les années 1950 et 1960. De forme juridique variable (fondation, SARL, association, etc.), ils sont financés par les pouvoirs publics (Bund et Länder) mais autonomes dans leur gestion. Leur mission est de réaliser une recherche publique à moyen et long terme, entretenant un certain équilibre entre recherche fondamentale, recherche dite " de prévoyance " car touchant à des domaines d'intérêt public prioritaires (climat, environnement, santé) et, dans certains champs de recherche, applications à un stade préindustriel. Les recherches menées dans les centres Helmholtz ont ainsi pour mission de résoudre des problèmes complexes qui mobilisent des moyens importants en termes financiers, de compétences scientifiques et d'équipement (ces centres construisent et gèrent les très grands instruments) et appellent par nature des coopérations interdisciplinaires.

Le FZJ jouit d'une reconnaissance internationale forte comme en atteste le nombre élevé de chercheurs étrangers accueillis (800 en 2003, d'une cinquan-
FZJ : des recherches pluridisciplinaires reconnues 
taine de nationalités), II est par ailleurs le plus grand centre inderdisciplinaire allemand de recherche fondamentale et appliquée, dans les domaines suivants :

- structure de la matière : physique des surfaces, matériaux hybrides multifonctionnels, nanosciences, électronique moléculaire, physique de la matière condensée, physique nucléaire... (dépenses $2004: 93$ millions $€$ );

- énergie: technologies de conversion de l'énergie, photovoltaïque, physique des plasmas, développement de matériaux pour les piles à combustible, fusion nucléaire, sécurité nucléaire, technologie des réacteurs... (53 millions $€$ );

- technologies-clés : supercalculateurs, électronique nucléaire... (46 millions $€$ ) ;

- environnement : recherches sur eaux, airs, sols, plantes au sein de l'Institut chimie et dynamique de la géosphère (32 millions $€$ ) ;

- sciences du vivant : recherches sur le cerveau et le cœur, chimie des radionucléides, biologie structurale et signalisation cellulaire (22 millions $€$ ), biotechnologies ( 7 millions $€$ ).

Une grande richesse en infrastructures et équipements

Il faut dire que le FZJ dispose, aux côtés d'un fort potentiel scientifique, de conditions expérimentales particulièrement favorables : un synchrotron à protons de $2,5 \mathrm{GeV}, 4$ cyclotrons, un réacteur nucléaire à neutrons, un réacteur à fusion nucléaire, une chambre de simulation atmosphérique, l'Institut John von Neumann for Computing (simulation par ordinateur) et, depuis juillet 2004, un tomographe à résonance magnétique 4-TESLA et un super-calculateur d'une capacité de 8,9 Téraflops. Le centre de Jülich abrite également l'Institut d'enzymologie de l'université Heinrich-Heine de Düsseldorf, ainsi qu'une partie des installations de médecine nucléaire du CHU de Düsseldorf, qui bénéficient ainsi de la proximité des équipements et du potentiel scientifiques.

\section{Réorganisation des structures institutionnelles de la recherche : l'exemple des centres Helmholtz}

Le débat récurrent au cours des années 1990 sur une meilleure allocation des financements publics, en particulier pour les grands organismes de recherche parfois accusés d'immobilisme, de même que les recommandations du Conseil des Sciences (Wissenschaftsrat) constatant un fort cloisonnement entre les centres Helmholtz, ont finalement abouti à une réforme stratégique engagée à l'automne 2001. Elle consacre l'abandon d'un mode de financement institutionnel au profit d'un financement pluriannuel ( 5 ans en règle générale) sur des thématiques de recherche (programmorientierte Förderung).

Les lignes directrices (priorités thématiques, cadre financier, principes généraux d'évaluation, éventuellement aussi objectifs et critères d'évaluation) sont élaborées par le Bund et les Länder réunis au sein d'un Comité des financeurs, sur la base d'échanges avec les milieux scientifiques, économiques, le Sénat de la HGF et les centres. Six domaines prioritaires (Santé ; Transports, aéronautique et espace ; Energie ; Terre et environnement ; Structure des matériaux ; Technologies-clefs), déclinés en 30 programmes de recherche, ont ainsi été définis. Les équipes de recherche sont appelées à présenter des projets soumis à l'évaluation préalable d'une commission d'experts internationaux externes à la HGF. Cette évaluation, qui s'est échelonnée entre 2002 et 2004, est fonction de trois critères : la qualité scientifique, la pertinence stratégique et l'adéquation entre objectifs et moyens mobilisés. Elle est censée attester la qualité des équipes et permettre un meilleur positionnement des centres dans la recherche allemande et européenne. Elle débouche sur des recommandations concrètes de financement pour la durée des programmes, soumises aux organismes financeurs (et sous réserve du vote du budget au parlement). Ce mode de financement concerne dès 2005 l'ensemble des six domaines.

L'objectif visé par cette réforme est de mieux exploiter l'énorme potentiel scientifique des centres par une combinaison de coopération renforcée entre instituts souvent très spécialisés et de concurrence entre centres et programmes pour l'obtention des financements. Avec la concentration des moyens sur certaines activités de recherche, chaque centre Helmholtz est ainsi appelé à confirmer sa spécialisation.

Ces nouvelles modalités de financement ont suscité initialement un certain scepticisme au sein des centres. S'il est admis généralement que la division en programmes aide les organismes financeurs à garder une meilleure visibilité sur l'utilisation des moyens alloués, on sait aussi que la réduction du financement institutionnel au profit des programmes est une mesure fortement structurante et qui bénéficie à certaines priorités de recherche. Mais les expériences dans les laboratoires depuis 2003 semblent aller dans le sens d'une plus forte transparence du financement, de liens resserrés entre centres, et contribuent finalement à évacuer certaines craintes. Les bons résultats des évaluations ont par ailleurs donné une publicité favorable à des institutions qui avaient dû subir des critiques récurrentes en raison de leur statut de grands organismes publics. Enfin, la mise en œuvre de la réforme s'est accompagnée de deux signaux forts destinés à calmer l'inquiétude des scientifiques. Les centres peuvent aujourd'hui disposer librement d'une enveloppe financière équivalant à $20 \%$ du montant des financements accordés sur programme, qui doit laisser les équipes libres de développer de nouvelles thématiques. Par ailleurs, un suivi régulier de la structure de l'emploi doit permettre d'analyser l'impact du nouveau mode de financement sur l'équilibre entre emplois fixes et contrats limités à la durée des programmes.

Reste la question, encore ouverte, de l'évaluation des programmes selon une procédure de controlling complexe : et beaucoup de pointer les difficultés d'une procédure de contrôle ex post reposant sur des indicateurs définis d'après une stratégie ex ante. 
Le financement de base est traditionnellement assuré par le Bund et le Land de NRW (247,08 millions $€$ en 2003), auquel s'ajoutent les revenus issus de contrats de recherche et de ressources diverses. Le Land finance par ailleurs à lui seul l'intégralité des recherches sur les biotechnologies. Des mesures exceptionnelles de régulation budgétaire ont particulièrement affecté le $\mathrm{FZJ}$ en 2003, alors même que le financement institutionnel basculait progressivement vers un financement par projets présentés par les équipes dans le cadre de priorités thématiques et soumis à une évaluation ex ante. Mais l'Etat fédéral s'est entre temps déclaré prêt à accorder aux organismes scientifiques des garanties de financement jusqu'en 2010, selon des modalités qui restent encore à préciser : une hausse globale au moins égale à $3 \%$ par an (à ventiler entre domaines de recherche) reste donc envisagée, en dépit des efforts de consolidation budgétaire, dans le cadre d'un « Pacte pour la recherche et l'innovation ». En contrepartie, les organismes devront accepter de nouvelles mesures destinées à attester de l'efficacité et la qualité de leurs travaux.

Pour le FZJ, les résultats globalement très positifs des expertises ont été généralement perçus comme une confirmation des orientations et choix thématiques du centre. Une hausse sensible de crédits est ainsi annoncée jusqu'en 2008 pour les programmes des domaines Terre et environnement et Energie, alors que dans le même temps, la stagnation déjà programmée des budgets dans certains domaines comme la fusion et la sécurité nucléaires a été confirmée, contraignant les équipes concernées à redéfinir les priorités de leur activité. Le passage au nouveau mode de financement n'a donc pour l'heure pas conduit à un changement de cap majeur dans ce centre à forte orientation interdisciplinaire. Pour le FZJ, on peut donc davantage parler d'une réforme dans la continuité, même si l'expertise a mis en exergue quelques correctifs à apporter, comme la nécessité de concrétiser davantage encore la coopération entre centres Helmholtz. Au-delà, la mise en œuvre de la réforme constitue néanmoins en interne un enjeu organisationnel puisque le nouveau mode de financement implique une modification des procédures administratives et un fort investissement en temps: nouvelles procédures budgétaires, rédaction de compte rendus d'avancement des programmes à transmettre au Sénat de la HGF, etc.

Conformément à l'objectif politique d'une meilleure cohérence et complémentarité au sein du système de recherche, un des principaux enjeux de la réforme de la HGF est d'accroître la coopération entre organismes scientifiques et, en premier lieu, avec les universités. Désireux de tirer profit au mieux de son potentiel scientifique (voir REA 62/03), le Land de NRW confirme cette priorité et encourage la constitution d'équipes de recherche capables d'accéder à un niveau de reconnaissance internationale par la constitution de réseaux coordonnant au mieux les capacités de recherche. Ce principe n'est pas nouveau pour le FZJ qui, depuis sa création, multiplie avec les universités des collaborations, dont la plupart se cristallisent autour de l'utilisation des grands instruments (le site www.fz-juelich.de reprend les nombreux projets en cours). Entretenant des liens directs avec 360 établissements universitaires en Allemagne et à l'étranger, le centre de Jülich se présente dès lors comme une plateforme de compétences rapprochant universités et équipes extra-universitaires. En 2003, le montant des prestations de recherche effectuées pour le compte du FZJ dans des établissements universitaires (allemands ou à l'étranger) a ainsi franchi les 3 millions $€$. Le FZJ participe par ailleurs à de nombreux programmes de coopération scientifique financés par la Deutsche Forschungsgemeinschaft (notamment dans 11 unités de recherche spécialisée: Sonderforschungsbereiche, en NRW). II est présent dans plusieurs centres régionaux et réseaux nationaux de compétences, qui organisent la coopération entre acteurs dans des domaines stratégiques, en tant que membre ou coordonnateur, comme dans le cas du réseau Kompetenznetzwerk Brennstoffzelle NRW (voir la plateforme
Un financement en cours de réforme

Une expertise favorable des travaux menés au FZJ

L'expérience d'une activité scientifique en réseau 
Une implication renforcée dans la formation scientifique

Des liens suivis avec les milieux économiques

Du partenariat à la valorisation www.kompetenznetze.de, depuis peu en français). Par ailleurs, la création de 13 instituts virtuels par le FZJ, financés par un fonds spécifique de la présidence de la HGF, constitue une nouvelle forme de coopération avec les universités, souple et à dimension variable, susceptible de mutualiser des compétences sur des objets de recherche complexes et souvent très spécifiques.

En dépit de ces formes multiples de coopération institutionnelle, le système scientifique allemand continue de souffrir, selon de nombreux observateurs, d'une trop forte étanchéité entre contenus de l'enseignement universitaire et recherche de pointe menée au sein des grands organismes. La HGF invite donc les centres à contribuer plus activement à la qualité de la formation scientifique, par exemple en favorisant les doubles nominations de professeurs. Cette pratique, peu systématique en Allemagne (en dehors des Instituts Max-Planck), est pourtant répandue depuis les années 1960 au sein du FZJ, qui fait ici figure de pionnier. Aujourd'hui, le centre compte une quarantaine de professeurs d'université y dirigeant simultanément un institut de recherche, et c'est globalement un cinquième des scientifiques de Jülich qui exerce une activité d'enseignement dans une trentaine d'établissements du supérieur. Dans le domaine de la formation des jeunes chercheurs, la coopération avec les universités devrait par ailleurs prendre une dimension nouvelle avec l'accord conclu en juillet 2004 entre les présidents de la Conférence des présidents d'université et la HGF : comités de soutenance mixtes et collèges doctoraux conjoints viendront s'ajouter aux unités de recherche spécialisée et aux groupes communs de jeunes chercheurs (Helmholtz-Hochschulnachwuchsgruppen) déjà en place.

Caractéristiques du système scientifique allemand, les liens entretenus entre institutions productrices de savoir et entreprises (y compris les PME) fonctionnent traditionnellement de manière satisfaisante. Les centres Helmholtz, notamment depuis leur réforme, ont toutefois vocation à renforcer les liens entre recherche et développement technologique, en particulier dans des domaines complexes, aux perspectives de développement encore incertaines ou mobilisant des moyens importants. Priorité gouvernementale absolue, l'innovation dans l'industrie et, in fine, la compétitivité du pays, passe en effet par le développement de nouvelles technologies susceptibles d'aboutir à de nouveaux marchés, et les grands centres publics sont appelés à jouer un rôle majeur dans le développement de domaines (comme les sciences de la matière à Jülich) ou technologies-clefs. Les infrastructures scientifiques de haut niveau servent dès lors de moteur pour le développement technologique et ouvrent la voie à des coopérations industrielles.

Le bureau de transfert de technologie du FZJ recense ainsi environ 2700 partenariats en 30 ans, qu'il s'agisse de contrats industriels, services et coopérations de R\&D, ou encore de licences de brevets, plus particulièrement dans les domaines de la photovoltaïque, de l'énergie, des biotechnologies ou des composants. Les entreprises Bayer AG, Degussa-Hüls, DSM Biotech GmbH, RWE Solar $\mathrm{GmbH}$, Siemens AG ou encore Philips et Osram, figurent parmi les principaux partenaires du centre. Une centaine de demandes de brevet en moyenne est déposée chaque année par le FZJ, et les exemples de valorisation sont nombreux ; en 2003 par exemple, un contrat de licence a été conclu avec la société Berlin Heart AG pour un procédé inédit de pompes cardiaques avec rotor magnétique. L'activité de transfert tend d'ailleurs à être systématisée avec plusieurs initiatives de l'association Helmholtz pour donner une meilleure visibilité aux résultats des recherches: création d'un guichet unique (One Stop Shop Ascenion) qui valorise les résultats en sciences du vivant, ouverture d'une banque de données des résultats scientifiques (www.helmholtz-experten.de) ou encore nouveaux modèles de partenariat industriel. Le FZJ propose également des initiatives spécifiques, comme la création d'un « Centre de formation et de démonstration sur les piles à combustibles », soutenu par le BMWA et le Land de NRW, en liaison avec les chambres de commerce et d'industrie et des fédérations professionnelles : l'objectif est d'aider à diffuser auprès des entreprises 
un savoir théorique et pratique et à contribuer à la qualification de personnels (techniciens, ingénieurs) qui faciliteront l'implantation de cette technologie sur les marchés.

Mais les grands centres Helmholtz, et plus particulièrement le centre de Jülich, ont aussi la fonction de servir de point d'ancrage pour la participation allemande à des programmes internationaux. Des coopérations régulières sont menées avec près de 500 partenaires internationaux en Europe, aux USA, en Russie et en Chine, permettant au FZJ de revendiquer un rôle intégrateur par ses compétences en ingénierie de projet et sa capacité à fédérer des partenaires de recherche et des industriels autour de programmes complexes. Le FZJ peut ainsi faire valoir de multiples participations à des projets européens, et plusieurs coordinations, comme le projet intégré REAL SOFC (pile à combustible) ou le réseau d'excellence Soft Matter Composites dans le cadre du $6^{\mathrm{e}}$ PCRD. En 2003, 149 projets européens étaient en cours (soit 10,3 millions $€$ par an) et 163 ont été déposés avec un taux de succès supérieur à la moyenne européenne. Le FZJ est par ailleurs un des huit partenaires du nouveau projet européen DEISA piloté par l'IDRIS-CNRS (Orsay), dont l'objectif est de créer une super-infrastructure européenne de calcul distribué de très haute performance, reliant entre elles les plateformes de calcul nationales.

Le FZJ, comme 5 autres centres Helmholtz, a la particularité d'héberger le Projektträger Jülich (PTJ). Cette unité autonome au sein du FZJ est un des treize 'porteurs de projets' (contre une trentaine il y a quelques années), ces structures qui sont mandatées par les autorités publiques pour les décharger de la gestion administrative et financière des programmes de recherche et technologiques. Le PTJ est le deuxième Projektträger en termes d'importance (après celui sis au sein du Centre de recherches aérospatiales PT-DLR), avec 320 collaborateurs répartis entre le site de Jülich et les deux antennes de Berlin et Rostock. Toutefois, bien que situé au sein du FZJ et alors même que leurs histoires sont étroitement liées, le PTJ concentre son activité exclusivement sur les demandes extérieures et ne gère aucune des participations du centre de recherche à des programmes nationaux ou internationaux. Plus récemment créé auprès du FZJ, le Projektträger Energie, Technologie, Nachhaltigkeit (ETN) se consacre pour sa part exclusivement à la gestion de programmes pour le Land de NRW.

Le PTJ travaille pour le compte de trois ministères fédéraux (BMBF, BMU, BMWA) et de plusieurs Länder: gestion des programmes de biotechnologies du Bade-Wurtemberg, de tous les programmes du Mecklembourg, du programme "Neue-Werkstoffe in Bayern », et de certains programmes de soutien à l'innovation de NRW. En 30 ans d'existence, le PTJ a ainsi géré plus de 18000 projets de recherche pour un montant de 10 milliards $€$. Dans certains domaines (l'énergie en particulier), il propose également une expertise scientifique interne (50\% de ses collaborateurs sont des scientifiques) ou externe et contribue à la planification des priorités de recherche, la sélection des projets et à l'évaluation des programmes. Ses champs de compétences et d'expertise recouvrent les biotechnologies, le développement durable, l'énergie, les matériaux ou encore les recherches sur les milieux polaires, sous-marins et sur les techniques maritimes. Le PTJ est également mandaté pour le pilotage de programmes transversaux dans les domaines de la valorisation (initiative EXIST pour la création d'entreprises innovantes issues des universités, programme Verwertungsoffensive de mise en place de structures de valorisation chargées de gérer les brevets des universités ; programme FUTOUR 2000 pour la création d'entreprises technologiques dans les nouveaux Länder), ou du développement économique basé sur l'innovation (programme InnoRegio pour promouvoir des bassins d'innovation et de croissance dans les Länder orientaux). Enfin, l'antenne berlinoise du PTJ fait office également de cellule d'information sur l'ensemble des programmes de soutien du BMBF et assure le relais avec les gestionnaires de programmes compétents.
Un partenaire important de la coopération internationale

Le Projektträger Jülich : une structure sise auprès du FZJ

Ingénierie de projets et prestations de services 
Un acteur fort de la coopération européenne
La dimension internationale est présente dans de nombreux programmes portés par le PTJ, qui représente par ailleurs le gouvernement fédéral auprès de l'Agence internationale de l'énergie. Le PTJ pilote également quelques programmes bilatéraux de l'Allemagne (Australie, Chine, Brésil, Canada, Israël ou Russie), bien que l'essentiel de la coopération bilatérale revienne au PT-DLR. Par ailleurs, le PTJ fait office de point de contact national pour les programmes de recherche relevant de quatre priorités thématiques du $6^{\mathrm{e}}$ PCRD ainsi que dans le cadre des activités de "Soutien aux politiques et anticipation des besoins scientifiques et technologiques " de I'Union européenne. Le gouvernement fédéral a en effet choisi de faire des 'porteurs de projet' les points de contacts nationaux pour les programmes communautaires : ces structures déjà en place offrent la garantie d'une bonne réactivité aux thématiques de recherche, une grande proximité avec le milieu scientifique et la compétence du suivi de grands projets. Ils favorisent par ailleurs une meilleure articulation entre programmes nationaux de soutien et programmes européens, ainsi qu'une meilleure intégration des partenaires allemands. Le PTJ participe de ce fait à la gestion de plusieurs projets européens et, depuis peu, actions ERA-NET, en tant que partenaire ou coordonnateur (projet intégré BRITTA, action coordonnée Hy-Co ERA NET).

ÉLEMENT DE LA STRATEGIE POLITIQUE DE MODERNISATION des organismes de recherche, la réforme en cours des centres Helmholtz s'inscrit elle-même dans une double logique de soutien à l'innovation et à la compétitivité économique des territoires. Cette réforme, qui consacre le passage à un financement de priorités de recherche après évaluation, exige des centres une flexibilité renforcée pour réagir aux appels d'offre, et les incite à trouver un équilibre entre compétition pour les financements et coopération dans les activités de recherche. Principal centre interdisciplinaire en Allemagne, le centre de recherche de Jülich considère aujourd'hui cette réforme davantage comme la confirmation d'un cap déjà suivi. II joue en effet de longue date un rôle privilégié au sein de réseaux de recherche ainsi que dans le couplage des activités entre organismes scientifiques publics et milieux économiques. Au regard de l'objectif prioritaire en Europe de conduire des coopérations scientifiques à large échelle, le FZJ figure dès lors comme un partenaire capable de mobiliser un fort potentiel. II faut dire que le système de recherche allemand peut faire valoir une forte expérience et capacité de management de programmes complexes, que ce soit au sein même des centres de recherche ou au sein des entités autonomes qu'ils hébergent.

\section{Indications bibliographiques}

BUNDESMINISTERIUM Für BILDUNG UNd Forschung (BMBF), Bundesbericht Forschung 2004, et Chance für Deutschland und Europa. Das 6. Forschungsrahmenprogramm (www.bmbf.de)

FORSCHUNGSZENTRUM JÜLICH, Jahresbericht 2003

FORSCHUNGSZENTRUM JÜLICH, Kompetenz für die Zukunft. Einrichtungen der Forschung, Entwicklung und Beratung (www.fz-juelich.de)

FORSCHUNGSZENTRUM JüLICH, Researchers for Tomorrow. Research Centre Jülich and its cooperations with universities, mars 2004

HeLmholtz-Gemeinschaft, Helmholtz-Gemeinschaft 2004. Programme - Zahlen - Fakten, et Überblick über die programmorientierte Förderung der Helmholtz-Gemeinschaft (état au 21-05-2003)

ProJeKTTRÄGER JüLICH, Geschäftsbericht 2003

Technologiefolgenabschätzung - Theorie und Praxis, n 1, mars 2003. 